\title{
Cafeína e performance em exercícios anaeróbios
}

\author{
Leandro Ricardo Altimari ${ }^{1,2,3}$, Antonio Carlos de Moraes ${ }^{1,2}$, Julio Tirapegui ${ }^{13^{*}}$, \\ Regina Lúcia de Moraes Moreau ${ }^{4}$
}

\begin{abstract}
${ }^{1}$ Grupo de Estudo e Pesquisa em Metabolismo, Nutrição e Exercício. Centro de Educação Física e Desportos, Universidade Estadual de Londrina, ${ }^{2}$ Departamento de Ciência do Esporte, Faculdade de Educação Física, Universidade de Campinas, ${ }^{3}$ Departamento de Alimentos e Nutrição Experimental, Faculdade de Ciências Farmacêuticas, Universidade de São Paulo, ${ }^{4}$ Departamento de Análises Clínicas e Toxicológicas, Faculdade de Ciências Farmacêuticas, Universidade de São Paulo
\end{abstract}

\footnotetext{
*Correspondência:

J. Tirapegui

Departamento de Alimentos e Nutrição

Experimental

Faculdade de Ciências Farmacêuticas Universidade de São Paulo Av. Prof. Lineu Prestes, 580, Bloco 14 05508-900 - São Paulo, SP - Brasil E-mail: tirapegu@usp.br
}

O efeito ergogênico da cafeina sobre a performance em exercícios fisicos anaeróbios ainda não esta claro, da mesma forma que os mecanismos de ação envolvidos nesse tipo de esforço físico. As teorias que têm tentado explicar o efeito ergogênico da cafeína durante o exercício físico anaeróbio esta relacionada a efeito da cafeina em alguma porção do sistema nervoso central (SNC), e a propagação dos sinais neurais entre o cérebro e a junção neuromuscular, e também ao efeito da cafeina sobre o músculo esquelético, facilitando a estimulação-contração do músculo esquelético. Alguns estudos têm indicado aumento da força muscular acompanhado de maior resistência à instalação do processo de fadiga muscular após a ingestão de cafeína. Sugerese que isso ocorra muito mais pela ação direta da cafeína no SNC do que pela sua ação em nível periférico. Com relação aos exercícios máximos e supramáximos de curta duração, os estudos têm-se demonstrado controversos, embora a maior parte indique que a cafeina parece melhorar significativamente a performance em exercícios máximos de curta duração $(<5 \mathrm{~min})$, quando não precedidos por exercícios submáximos prolongados. Entretanto, esses resultados necessitam de confirmação, assim como de maior esclarecimento quanto aos mecanismos de ação da cafeina nesses tipos de esforços.
Unitermos

- Cafeína

- Ergogênico

- Performance

- Exercícios anaeróbios

\section{INTRODUÇÃO}

Na busca do sucesso esportivo de alto nível, treinadores, nutricionistas, médicos e cientistas têm lançado mão de inúmeros recursos ergogênicos no intuito de potencializar a performance atlética ou atenuar os mecanismos geradores de fadiga de seus atletas (Maughan, 1999; Juhn, 2003; Maughan, King, Lea, 2004).

A fadiga é apontada como fator limitante da performance atlética e constitui um fenômeno complexo 
ou até mesmo um conjunto de fenômenos de interação simultânea com diferentes graus de influência, dependendo da natureza do exercício físico (Davis, Bailey, 1997). No processo de instauração da fadiga, deve ser considerado não apenas o componente periférico (Fitts, 1994), mas também o componente central, que por sua vez tem recebido crescente atenção dos pesquisadores (Rossi, Tirapegui, 1999).

Neste sentido, a utilização de alguns suplementos nutricionais e substâncias com potencial ergogênico tem se mostrado eficiente por retardar o aparecimento da fadiga e aumentar o poder contrátil do músculo esquelético e/ou cardíaco, aprimorando, portanto, a capacidade de realizar trabalho físico, ou seja, a performance atlética (Gomes, Tirapegui, 2000; Maughan, 2002; Pipe, Ayotte, 2002; Juhn, 2003; Coyle, 2004).

Desse modo, a cafeína tem sido utilizada como substância ergogênica de forma aguda, previamente à realização de exercícios anaeróbios (alta intensidade e curta duração), com o intuito de protelar a fadiga e, conseqüentemente, aprimorar a performance. Embora a maioria dos estudos não seja conclusiva em relação aos mecanismos responsáveis pelos efeitos da cafeína no metabolismo anaeróbio, os achados até o presente momento têm apontado a cafeína como um possível agente ergogênico em exercícios dessa natureza (Altimari et al., 2000; Bucci, 2000; Graham, 2001A; Paluska, 2003).

\section{CAFEÍNA}

A cafeína (1,3,7-trimetilxantina) é um derivado da xantina, quimicamente relacionada com outras xantinas: teofilina (1,3-dimetilxantina) e teobromina $(3,7$ dimetilxantina). Elas se diferenciam pela potência de suas ações farmacológicas sobre o sistema nervoso central (SNC) (Figura 1) (George, 2000).

Esta substância está presente em vários produtos consumidos diariamente, como o guaraná, o mate, o chocolate, o café, alguns refrigerantes e chás (Clarkson, 1993; Slavin, Joensen, 1995; Barone, Roberts, 1996). Esta também pode ser encontrada em alguns medicamentos como agente antagonizador do efeito calmante de certos fármacos (Spriet, 1995; Sinclair, Geiger, 2000). A Tabela I apresenta as principais fontes de cafeína na dieta.

TABELA 1 - Principais fontes de cafeína na dieta ${ }^{\mathrm{a}}$

\begin{tabular}{lc}
\hline Produto & $\begin{array}{c}\text { Conteúdo de cafeína } \\
\text { (mg) }\end{array}$ \\
\hline Café (xícara 150 mL) & 85 \\
$\quad$ Torrado e moído & 60 \\
$\quad$ Instantâneo & 3 \\
$\quad$ Descafeinado & 30 \\
Chá (xícara 150 mL) & 20 \\
$\quad$ Folhas & \\
Instantâneo & 6 \\
Chocolate & 20 \\
$\quad$ Barra de chocolate ao leite $(29 \mathrm{~g})^{\mathrm{b}}$ & 4 \\
$\quad$ Barra de chocolate escuro $(29 \mathrm{~g})^{\mathrm{b}}$ & $5-20$ \\
$\quad$ Achocolatados $(180 \mathrm{~mL})$ & 18 \\
$\quad$ Outros produtos $(100 \mathrm{~g})$ & 46 \\
Refrigerantes tipo cola $(180 \mathrm{~mL})$ & 38 \\
$\quad$ Coca-cola (lata $360 \mathrm{~mL})^{\mathrm{c}}$ & \\
Pepsi-cola (lata $360 \mathrm{~mL})^{\mathrm{c}}$ &
\end{tabular}

Adaptado de aarone, Roberts (1996); 'Ellenhorn's Medical Toxicology (1997); 'Harland (2000).

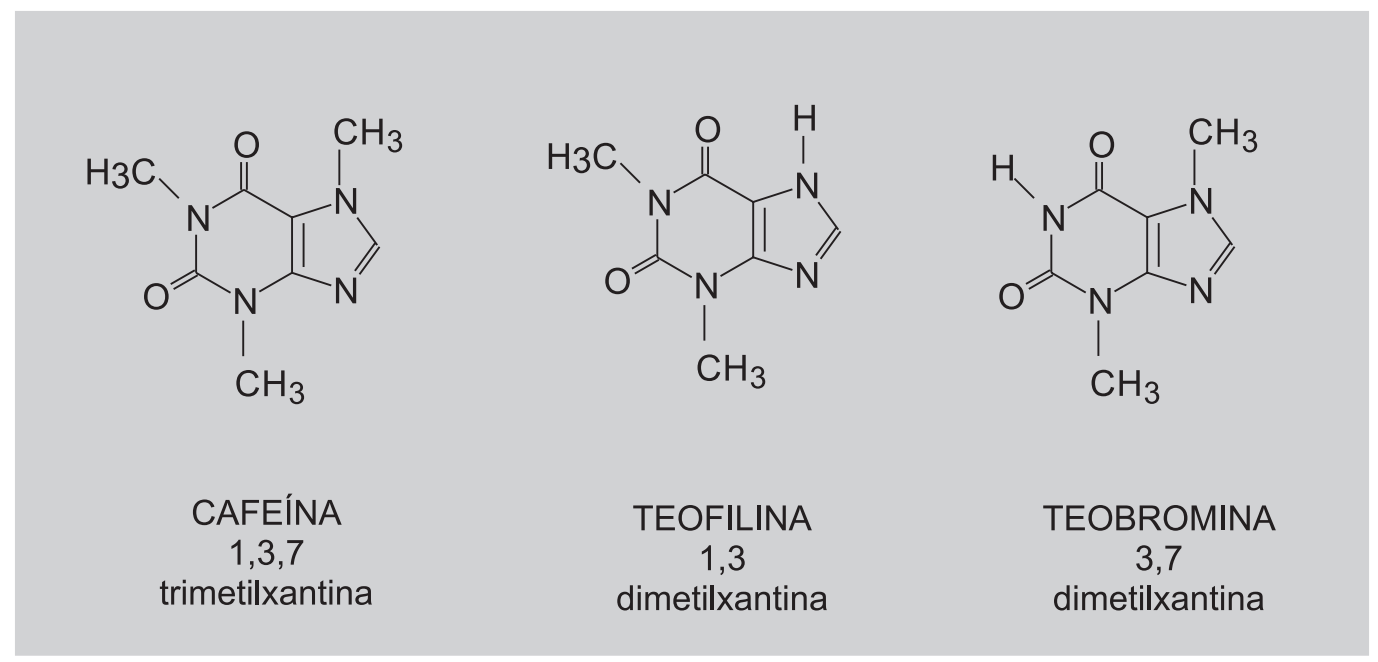

FIGURA 1 - Estrutura química da cafeína e metilxantinas relacionadas. 
Nos últimos anos a cafeína tem sido alvo de inúmeros estudos envolvendo exercícios físicos com características aeróbias (moderados de média e longa duração), sendo que os achados até o presente momento têm demonstrado que esta substância é um eficiente agente ergogênico em exercícios físicos aeróbios (Braga, Alves, 2000; Altimari et al., 2001; Graham, 2001b; Juhn, 2002; Spriet, Gibala, 2004).

A cafeína esteve incluída na lista de substâncias proibidas pela World Anti Doping Agency (WADA/2004) na classe de estimulantes (A) até final do ano de 2003. Entretanto, mais recentemente a retirou a cafeína da lista de substâncias proibidas, incluindo esta em um programa de monitoramento, o qual será feito por meio de acompanhamento na incidência de detecção do uso de cafeína pelos atletas.

\section{ABSORÇÃO, BIOTRANSFORMAÇÃO E EXCREÇÃO}

A cafeína é uma substância absorvida rapidamente e eficientemente, através do trato gastrintestinal após administração oral. A mesma parece não afetar as funções gastrintestinais quando ingerida de forma conjugada a diferentes soluções líquidas, como carboidrato e água (Sinclair, Geiger, 2000; Van Nieuwenhoven, Brummer, Brouns, 2000). Esta substância pode alcançar concentração máxima na corrente sangüínea entre 15 e 120 minutos após a sua ingestão (Sinclair, Geiger, 2000). Vale ressaltar que grande parte dos estudos utiliza o intervalo de 60 minutos entre a ingestão de cafeína e o início do exercício físico, uma vez que este parece ser o tempo em que se observa a maior concentração de cafeína na corrente sangüínea após ingestão (McLean, Graham, 1998; Graham, 2001A; Graham, 2001B).

A administração desta substância pode ser feita de diversas formas, dentre as quais destacamos a administração oral, intraperitoneal, injeções subcutânea ou intramuscular e também através da aplicação de supositórios (Wang, Lau, 1998; Sinclair, Geiger, 2000; Graham, 2001b). Sua ação pode atingir todos os tecidos, pois o seu transporte é feito via corrente sangüínea, sendo posteriormente degradada e excretada pela urina (Clarkson, 1993; Spriet, 1995; Sinclair, Geiger, 2000).

A biotransformação da cafeína ocorre em maior proporção no fígado, no qual existe maior concentração de citocromo P450 1A2, enzima responsável pelo metabolismo desta substância (Kalow, Tang, 1993; Sinclair, Geiger, 2000). Ocorre primeiramente a remoção do grupo metil nas posições 1 e 7, catalisada pelo citocromo P450 1A2, o que possibilita a formação de três grupos metilxantina
(Ferdholm, 1985). Em humanos, a maior parte do metabolismo da cafeína ocorre pela mudança na posição do grupo metil $1,3,7$ possibilitando predominância $(84 \%)$ na forma de paraxantina (1,7-dimetilxantina), seguida de teofilina (1,3-dimetilxantina) e de teobromina $(3,7$ dimetilxantina) (Sinclair, Geiger, 2000).

Embora a maior parte da biotransformação da cafeína ocorra no fígado, outros tecidos, incluindo o cérebro e o rim, também participam nesse processo, pelo importante papel na produção de citocromo P450 1A2 (Goabduff et al., 1996). A cafeína é lentamente catabolisada, apresentando meia-vida de 4 a 6 horas (McLean, Graham, 1998).

Apesar de apenas pequena quantidade de cafeína ser excretada $(0,5$ a $3 \%)$, sem alteração na sua constituição química, sua detecção na urina é relativamente fácil (Clarkson, 1993; Graham, 2001a). Vale ressaltar que alguns fatores como a genética, a dieta, o uso de alguma droga, o sexo, o peso corporal, o estado de hidratação, o tipo de exercício físico praticado e o consumo habitual de cafeína, podem afetar seu metabolismo e, conseqüentemente, influenciar na quantidade urinária total excretada (Duthel et al., 1991; Spriet, 1995; Sinclair, Geiger, 2000).

\section{MECANISMOS DE AČ̃̃O E PERFORMANCE EM EXERCÍCIOS ANÁERÓBIOS}

Acredita-se que a cafeína possua mecanismos de ação central e periférica que podem desencadear importantes alterações metabólicas e fisiológicas, as quais parecem melhorar a performance atlética (Graham, Rush, Van Soeren, 1994; Graham, 2001a; Graham, 2001b). Todavia o seu efeito ergogênico é ainda bastante controverso, visto que aparentemente outros mecanismos podem estar associados à sua ação melhorando, a performance em diferentes tipos de exercício (Spriet, 1995).

Dessa forma, uma primeira teoria que pode tentar explicar o efeito ergogênico da cafeína durante o exercício físico anaeróbio, esta relacionada ao efeito direto da cafeína em alguma porção do sistema nervoso central, afetando a percepção subjetiva de esforço e/ou a propagação dos sinais neurais entre o cérebro e a junção neuromuscular (Spriet, 1995; Davis et al., 2003). Acredita-se ainda que a ação estimulante da cafeína no SNC envolve a estimulação do sistema nervoso simpático, aumentando a liberação e, conseqüentemente, a ação das catecolaminas (Yamada, Nakazato, Ohga, 1989; Rachima-Maoz, Peleg, Rosenthal, 1998). Contudo, essa hipótese é ainda extremamente especulativa, haja vista as grandes limitações que envolvem esse tipo de investigação. 
Uma segunda teoria pressupõe o efeito direto da cafeína sobre o músculo esquelético. As possibilidades incluem: alteração de íons, particularmente sódio e potássio; inibição da fosfodiesterase (PDE), possibilitando um aumento na concentração de adenosina monofosfato cíclica (AMPc); efeito direto sobre a regulação metabólica de enzimas semelhantes às fosforilases (PHOS); e aumento na mobilização de cálcio através do retículo sarcoplasmático e, conseqüentemente, aumento dos níveis intracelulares de cálcio nos músculos, facilitando a estimulação-contração do músculo esquelético, aumentando a eficiência da contração (Spriet, 1995; Sinclair, Geiger, 2000; Davis et al., 2003). Essas possibilidades têm sido levantadas a partir de investigações in vitro, em que altas concentrações de cafeína (10 a 80 mM) são empregadas na tentativa de demonstrar seus efeitos (Issekutz, 1984; Yamada, Nakazato, Ohga, 1989). Entretanto, acredita-se que as concentrações de cafeína necessárias para inibir a PDE e a PHOS e, conseqüentemente, desencadear série de reações metabólicas são bem superiores às utilizadas nesses estudos (Spriet, 1995).

Aparentemente, a cafeína pode agir diretamente sobre o músculo, potencializando sua capacidade de realizar exercícios físicos de alta intensidade e curta duração (Lopes et al., 1983). A hipótese atualmente aceita para essa ocorrência estabelece que a cafeína age sobre o retículo sarcoplasmático aumentando sua permeabilidade ao cálcio, tornando este mineral prontamente disponível para o processo de contração muscular. Assim, é provável que a cafeína possa influenciar a sensibilidade das miofibrilas ao cálcio (Roy et al.,1994; Pinto, Tarnopolsky, 1997).

Segundo Pagala e Taylor (1998), o mecanismo de ação do cálcio induzido pela ação da cafeína parece agir de forma diferenciada nas fibras musculares do tipo I e II, visto que as fibras de contração lenta (tipo I) são mais sensíveis à ação da cafeína do que as fibras musculares de contração rápida (tipo II).

Poucos estudos têm procurado investigar os efeitos ergogênicos da cafeína sobre a performance em exercícios de alta intensidade e curta duração (força, velocidade e potência). Além disso, os resultados encontrados até o momento têm sido bastante controversos, impossibilitando conclusões mais definitivas a esse respeito.

As maiores dificuldades para interpretação dos resultados produzidos por esses estudos concentram-se nos diferentes delineamentos utilizados, nas diferentes doses de cafeína administradas, nas diferenças entre os protocolos experimentais, que muitas vezes combinam exercícios predominantemente aeróbios e anaeróbios, na falta de maior rigidez metodológica no controle de variáveis supos- tamente envolvidas no processo, dentre outras. Alguns estudos que procuraram investigar os possíveis efeitos deste ergogênico (cafeína) sobre a performance em exercícios de alta intensidade e curta duração são apresentados com mais detalhes na Tabela 2 .

Estudo conduzido por Lopes et al. (1983) não constatou qualquer efeito da suplementação de cafeína sobre a força muscular durante contrações voluntárias máximas (CVM) do músculo adutor do polegar. Nesse mesmo estudo verificou-se que, durante um esforço submáximo (50\% CVM), a administração de cafeína produz aumento na força somente quando a freqüência de estimulação é baixa $(10,20,30$ e $50 \mathrm{~Hz})$. Tal efeito foi observado tanto antes quanto após a instalação do estado de fadiga muscular. Esses resultados são indicativos de possível efeito ergogênico específico e direto da cafeína sobre o músculo esquelético, quando estimulado em baixas freqüências.

Roy et al. (1994), após analisarem a resposta dos músculos dorsi-flexores frente à estimulação elétrica em indivíduos sadios antes e após esforço submáximo (75 e $85 \%$ do $\left.\mathrm{VO}_{2} \max \right)$ em cicloergômetro, constataram que a administração aguda de cafeína retarda a fadiga muscular quando induzida por uma força tetânica de $100 \mathrm{~Hz}$.

Aumento significativo na força de contração máxima foi observado por Pinto e Tarnopolsky (1997), após a ingestão de cafeína tanto em homens quanto em mulheres submetidos a estimulação elétrica dos músculos dorsiflexores. Vale destacar que nesse estudo as mulheres apresentaram maior resistência à fadiga muscular. Em contra-partida, Hespel et al. (2002), também utilizando-se de estimulação elétrica, porém em atividade intermitente, não constataram melhora significativa na força máxima, no tempo de contração e tempo de relaxamento do músculo quadríceps após ingestão aguda de cafeína (60 minutos antes do esforço). Contudo, nesse mesmo estudo, quando a ingestão de cafeína foi realizada de forma crônica (por 3 dias seguidos), verificou-se melhora significativa na força máxima, no tempo de contração e tempo de relaxamento do músculo quadríceps.

Kalmar e Cafarelli (1999) investigaram o efeito da administração de cafeína sobre a função neuromuscular por meio de sinais eletromiográficos. Os autores verificaram aumento significativo nas contrações voluntárias máximas $(3,5 \%)$ e no tempo de execução das contrações até a instalação da fadiga muscular $(25,8 \%)$ do músculo vasto lateral. Para esses pesquisadores, a cafeína aumenta a ativação voluntária máxima pela sua ação direta sobre o sistema nervoso central (SNC), indicando que o mecanismo de ação periférica da cafeína atua em menor intensidade. 
TABELA II - Efeito ergogênico da cafeína sobre a performance em exercícios de curta duração e alta intensidade

\begin{tabular}{lccll}
\hline Investigadores & N & Sexo & População & $\begin{array}{c}\text { Dose de } \\
\text { Cafeína }\end{array}$ \\
\hline Anderson et al. (2000) & 8 & $\mathrm{M}$ & Treinados & $6 \mathrm{e} 9 \mathrm{mg} / \mathrm{kg}$ \\
& & & & \\
\hline Anselme et al. (1992) & 14 & $\begin{array}{c}10 \mathrm{M} \\
4 \mathrm{~F}\end{array}$ & Não-treinados & $250 \mathrm{mg}$ \\
& & 4 &
\end{tabular}

Bell et al. (2001)

$\begin{array}{lll}\text { Cicloergômetro à } & \text { Sim } & \begin{array}{l}\text { Aumento significativo no } \\ \text { tempo de exaustão, déficit } \\ 125 \% \text { do } \mathrm{VO}_{2} \text { máx }-\end{array} \\ \text { máximo déficit } & & \text { acumulado de } \mathrm{O}_{2} \text {, na } \\ \text { acumulado de } \mathrm{O}_{2} & & \begin{array}{l}\text { concentração de lactato, glicose } \\ \text { (MAOD) }\end{array}\end{array}$

\begin{tabular}{llllll}
\hline Bond et al. (1986) & 12 & $\mathrm{M}$ & Treinados & $5 \mathrm{mg} / \mathrm{kg}$ & $\begin{array}{l}6 \mathrm{RM} \text { de flexão e } \\
\text { extensão de joelho }\end{array}$
\end{tabular}

torque,

\begin{tabular}{lllll}
\hline Bruce et al. $(2000)$ & 8 & M & Treinados & 6 e $9 \mathrm{mg} / \mathrm{kg}$
\end{tabular}

\begin{tabular}{|c|c|c|c|c|}
\hline Collomp et al. (1990) & 7 & M & Não-treinados & $250 \mathrm{mg}$ \\
\hline Collomp et al. (1991) & 6 & $\begin{array}{l}3 \mathrm{M} \\
3 \mathrm{~F}\end{array}$ & Não-treinados & $5 \mathrm{mg} / \mathrm{k}$ \\
\hline Collomp et al. (1992) & 14 & $\mathrm{M}$ & $\begin{array}{l}7 \text { não-treinados } \\
\text { e } 7 \text { treinados }\end{array}$ & \\
\hline
\end{tabular}

\begin{tabular}{lllll}
\hline Doherty (1998) & 9 & $\mathrm{M}$ & Treinados & $5 \mathrm{mg} / \mathrm{kg}$
\end{tabular}
com 20 min de intervalo
2 tiros de 100 metros
F-V Exercise test com cargas progressivas $(2$, 4, 6, $8 \mathrm{~kg}$ ), cada qual com duração de 6 segundos.

velocidade de $30^{\circ}, 150^{\circ}$ e $300^{\circ} \mathrm{s}^{-1}$

Prova de remo 2.000 metros

$\operatorname{Sim}$

Não

Não se constatou aumento significativo nos picos de

na potência e no índice de fadiga

Aumento significativo no tempo de prova $(1,2 \%)$ e na potência média $(2,7 \%)$ em ambas as doses, 6 e $9 \mathrm{mg} / \mathrm{kg}$

Aumento não-significativo na resistência (9\%)

$100 \%$ do $\mathrm{VO}_{2}$ máx

Wingate $(30 \mathrm{~s})$

Não

Não se constatou aumento significativo no tempo de alcance da potência-pico e no trabalho total

Redução significativo no tempo de nado no $1^{\circ}$ e $2^{\circ}$ tiro de $100 \mathrm{~m}(2$ e $4 \%$, respectivamente)

Aumento significativo no tempo de exaustão e no déficit acumulado de $\mathrm{O}_{2}$

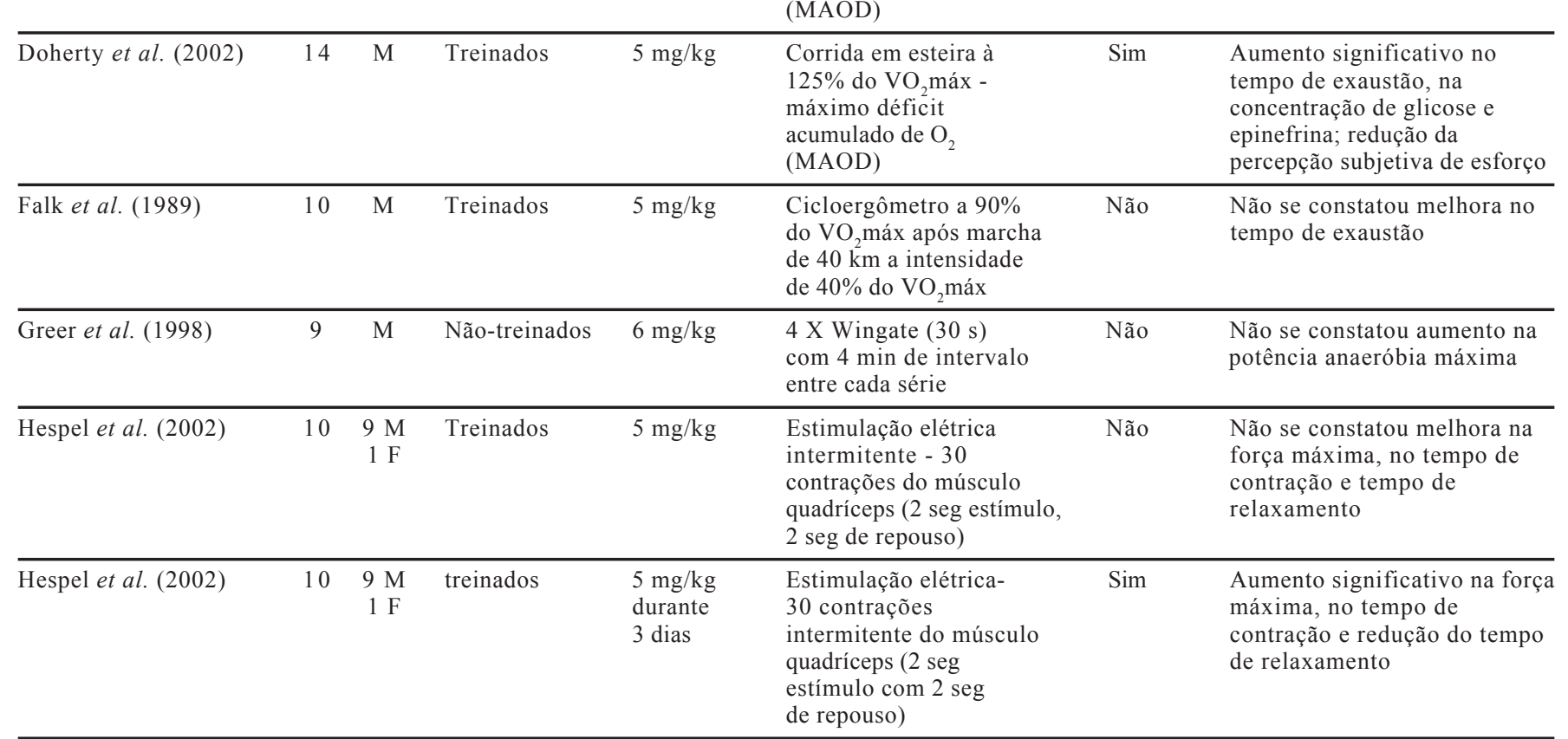


TABELA II - continuação

\begin{tabular}{lcclllll}
\hline Investigadores & N & Sexo & População & $\begin{array}{l}\text { Dose de } \\
\text { Cafeína }\end{array}$ & Tipo de teste & $\begin{array}{l}\text { Efeito } \\
\text { ergogênico? }\end{array}$ & Comentários \\
\hline Jackman et al. (1996) & 14 & $\begin{array}{c}11 \mathrm{M} \\
3 \mathrm{~F}\end{array}$ & $\begin{array}{l}\text { Não-treinados } \\
\text { e treinados }\end{array}$ & $6 \mathrm{mg} / \mathrm{kg}$ & $\begin{array}{l}\text { 2 sprints de } 2 \mathrm{~min} \\
\text { em cicloergômetro } \\
\text { sepa-rados por 6 min, } \\
\text { seguido de teste máximo } \\
\text { até exaustão }\end{array}$ & Sim & $\begin{array}{l}\text { Aumento significativo no } \\
\text { tempo de exaustão }\end{array}$ \\
\hline
\end{tabular}

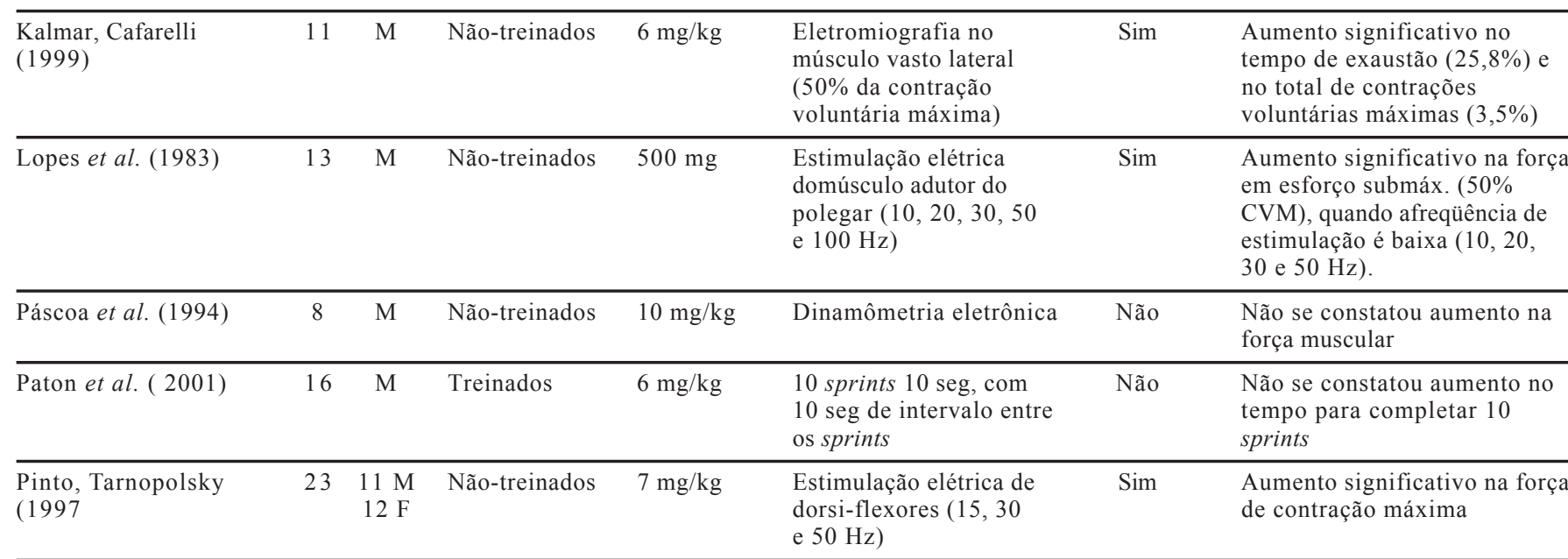

\begin{tabular}{llllllll}
\hline Plaskett, Cafarelli & 15 & M & Não-treinados & $6 \mathrm{mg} / \mathrm{kg}$ & Eletromiografia no & Sim & Aumento significativo $17,0^{\circ}$
\end{tabular}

no (2001)

$$
\text { (2001) }
$$

\begin{tabular}{|c|c|c|c|c|}
\hline Roy et al. (1994) & 5 & M & Treinados & $9 \mathrm{mg} / \mathrm{kg}$ \\
\hline
\end{tabular}

\section{músculo quadriceps $(50 \%$ da contração voluntária máxima)}

tempo de exaustão e redução da sensação de força durante os primeiros 10-20 seg. de contração

$\begin{array}{lll}\text { Estimulação elétrica de } & \text { Sim } & \text { Protelamento da fadiga } \\ \text { dorsi-flexores }(15,30,50 & & \text { muscular induzida a uma força } \\ \text { e } 100 \mathrm{~Hz}) \text { antes e após } & \text { tetânica de } 100 \mathrm{~Hz}\end{array}$

e $100 \mathrm{~Hz}$ ) antes e apó $85 \% \mathrm{VO}_{2}$ máx)

\begin{tabular}{|c|c|c|c|c|c|c|c|}
\hline Vanakoski et al. (1998) & 7 & M & Treinados & $7 \mathrm{mg} / \mathrm{kg}$ & $\begin{array}{l}3 \text { sprint de } 1 \text { min } \\
\text { em cicloergômetro com } \\
5 \text { min de intervalo entre } \\
\text { os sprints }\end{array}$ & Não & $\begin{array}{l}\text { Não se constatou melhora na } \\
\text { velocidade (rpm), manutenção } \\
\text { da velocidade (rpm) e no } \\
\text { trabalho total }\end{array}$ \\
\hline Walton et al. (2002) & 7 & M & Não-treinados & $6 \mathrm{mg} / \mathrm{kg}$ & $\begin{array}{l}\text { Eletromiografia em } \\
\text { dorsi-flexores ( } 3 \text { contrações } \\
\text { voluntárias máximas com } \\
1 \text { min de intervalo) }\end{array}$ & Sim & $\begin{array}{l}\text { Aumento significativo da } \\
\text { freqüência relativa de disparos } \\
\text { em unidades motoras do tibial } \\
\text { anterior }\end{array}$ \\
\hline Wemple et al. (1997) & 6 & $\begin{array}{l}4 \mathrm{M} \\
2 \mathrm{~F}\end{array}$ & Treinados & $8,7 \mathrm{mg} / \mathrm{kg}$ & $\begin{array}{l}\text { Cicloergômetro a } 60 \% \text { do } \\
\mathrm{VO}_{2} \text { máx durante } 180 \mathrm{~min} \\
\text { seguido de teste a } 80 \% \text { do } \\
\mathrm{VO}_{2} \text { máx }\end{array}$ & Não & $\begin{array}{l}\text { Não se constatou aumento no } \\
\text { tempo de exaustão; } \\
\text { manutenção da percepção } \\
\text { subjetiva de esforço }\end{array}$ \\
\hline Wiles et al. (1992) & 18 & $\mathrm{M}$ & Treinados & $150-250 \mathrm{mg}$ & Corrida de 1.500 metros & Sim & $\begin{array}{l}\text { Aumento significativo na } \\
\text { velocidade de corrida e redução } \\
\text { no tempo }\end{array}$ \\
\hline Willians et al. (1988) & 9 & M & Não-treinados & $7 \mathrm{mg} / \mathrm{kg}$ & Wingate $(15 \mathrm{~s})$ & Não & $\begin{array}{l}\text { Não se constatou aumento no } \\
\text { tempo de alcance da potência } \\
\text { pico, e no trabalho total }\end{array}$ \\
\hline Wyss et al. (1986) & 6 & $\mathrm{M}$ & Não-treinados & $\begin{array}{l}30,200 \\
\text { e } 300 \mathrm{mg}\end{array}$ & Wingate $(30 \mathrm{~s})$ & $\operatorname{Sim}$ & $\begin{array}{l}\text { Aumento significativo na } \\
\text { potência e na capacidade } \\
\text { anaeróbia após a ingestão } \\
\text { de } 300 \mathrm{mg}\end{array}$ \\
\hline
\end{tabular}

Plaskett e Cafarelli (2001) analisando a atividade eletromiográfica do músculo quadríceps após administração de cafeína constataram aumento significativo no tempo de exaustão $(17 \%)$ e redução da percepção de força durante os primeiros 10-20 segundos de contração. Mais recentemente, Walton, Kalmar e Cafarelli (2002) por meio 
de análise eletromiográfica dos músculos dorsi-flexores investigaram o efeito da ingestão de cafeína sobre a ativação de unidades motoras dos músculos tibiais anteriores e verificaram aumento significativo na freqüência relativa de disparos nas unidades motoras do tibial anterior.

Em contrapartida, Bond et al. (1986) investigaram o efeito da ingestão de cafeína sobre os níveis de força muscular avaliada em contrações voluntárias dinâmicas e não observaram melhorias significativos. Os autores atribuíram o resultado, possivelmente, aos baixos teores de cafeína utilizados. Da mesma forma, Páscoa, Alvim e Rodrigues (1994) não observaram aumento na força muscular em homens sadios, avaliados por meio de dinamometria eletrônica.

Williams et al. (1988) não verificaram aumento significativo na potência-pico máxima e na resistência muscular após a ingestão de cafeína em teste máximo de curta duração. Da mesma forma Collomp et al. (1991) não encontraram alteração significativa no pico da potência e no trabalho total em teste de Wingate atrelada ao uso dessa substância.

Greer, Maclean e Graham (1998) não encontraram qualquer efeito ergogênico que pudesse ser atribuído ao uso de cafeína na potência máxima em exercício máximo de curta duração. De forma semelhante, Collomp et al. (1990) não encontraram diferenças significativos no tempo de desempenho até a exaustão após a administração de cafeína.

Recentemente, em um estudo de campo, Paton, Hopkins e Vollebregt (2001) investigaram o desempenho de corredores durante exercício intermitente anaeróbio após a administração de cafeína e não constataram aumento no tempo de exaustão.

Por outro lado, Wyss et al. (1986) observaram aumento significativo na potência e na capacidade anaeróbia $(6,0 \%$ e $15,7 \%$, respectivamente) após a administração de dosagem de $300 \mathrm{mg}$ de cafeína. Do mesmo modo, Anselme et al. (1992) constataram melhora significativa de $7 \%$ na potência anaeróbia máxima durante exercício supramáximo de carga progressiva após suplementação com cafeína.

Falk et al. (1989) examinaram os efeitos da ingestão de cafeína no desempenho físico, logo após marcha de $40 \mathrm{~km}$ em pista a intensidade de $40 \%$ do $\mathrm{VO}_{2}$ máx. Os autores não constataram melhora significativo no tempo de desempenho até a exaustão. Nesse sentido, Wemple, Lamb e McKeever (1997) também não observaram melhora significativa na percepção de esforço, bem como no tempo de exaustão, após administração de cafeína em protocolo de exercício físico de 180 minutos a $60 \%$ do $\mathrm{VO}_{2}$ máx seguido por um teste máximo a $80 \%$ do $\mathrm{VO}_{2}$ máx.
Jackman et al. (1996) após submeterem um grupo de indivíduos treinados e não treinados a esforços intermitentes em cicloergômetro ( 2 esforços de 2 min a $100 \%$ do $\mathrm{VO}_{2}$ máx separados por 6 min), seguido por um teste máximo até a exaustão voluntária, concluíram que a ingestão de cafeína pode resultar em aumento da resistência muscular durante exercícios físicos intensos que levam à fadiga em até 5 minutos.

Mais recentemente, Doherty (1998) examinando o desempenho em corrida de alta intensidade (3-4 min), observou melhora significativa no débito máximo de oxigênio acumulado e no tempo de exaustão após ingestão de cafeína. Esses achados foram confirmados posteriormente utilizando-se do mesmo modelo de exercício (Doherty et al., 2002). Da mesma forma Bell, Jacobs e Ellerington (2001) constataram melhora significativa no débito máximo de oxigênio acumulado e no tempo de exaustão em cicloergômetro após ingestão de cafeína. Esses resultados tem implicações importantes, uma vez que a melhora do desempenho físico demonstrou estar relacionada a possível ação ergogênica da cafeína sobre a capacidade anaeróbia.

Estudo de campo realizado por Collomp et al. (1992) demonstrou redução significativa no tempo de nado nos 100 metros livres após a ingestão de cafeína. Em estudo similar, Wiles et al. (1992) verificaram que a ingestão de cafeína melhorou de forma significativa a velocidade e o tempo de corrida em uma prova de 1.500 metros. De forma semelhante Bruce et al. (2000) e Anderson et al. (2000) investigando o efeito de diferentes doses de cafeína $(6$ e $9 \mathrm{mg} / \mathrm{kg}$ ) em um grupo de remadores durante prova simulada de 2.000 metros, constataram redução significativa no tempo de prova. Segundo os autores, a melhora da performance após ingestão de cafeína foi determinada nos 500 metros iniciais de prova, indicando uma possível ação ergogênica desta substância sobre o metabolismo anaeróbio.

\section{CONSIDERAÇÕES FINAIS}

O efeito ergogênico da cafeína sobre a performance em exercícios físicos com características aeróbias (moderados de média e longa duração) tem sido envidenciado pela literatura. Contudo, o efeito desta substância sobre a performance anaeróbia (alta intensidade e curta duração) ainda não esta claro, da mesma forma que os mecanismos de ação envolvidos nesse tipo de esforço físico, indicando a necessidade de novas pesquisas com intuito de esclarecer a verdadeira ação desta substância sobre o metabolismo anaeróbio.

Alguns estudos têm indicado aumento da força muscular acompanhado de maior resistência à instalação 
do processo de fadiga muscular após a ingestão de cafeína. Entretanto, ainda não está totalmente claro qual o mecanismo de ação responsável pelo aumento da força muscular; todavia, sugere-se que isso ocorra muito mais pela ação direta da cafeína no SNC do que pela sua ação em nível periférico.

Quanto aos exercícios máximos e supramáximos de curta duração, os resultados têm-se demonstrado controversos. Embora a maioria dos estudos dessa natureza demonstre que a ingestão de cafeína melhora significativamente a performance em exercícios máximos de curta duração ( $<5$ minutos), o mesmo não se pode dizer com relação a tais exercícios quando precedidos por exercícios submáximos prolongados. Nesses casos, a performance atlética parece não sofrer qualquer alteração. Entretanto, esses resultados necessitam de confirmação, assim como de maior esclarecimento quanto aos mecanismos de ação da cafeína nesses tipos de esforços.

\section{ABSTRACT}

\section{Caffeine and performance in anaerobic exercise}

The ergogenic effects of caffeine on performance in anaerobic activites are not clear yet, so are the mechanisms involved in this type of physical effort. The theories that have been trying to explain the ergogenic effects of caffeine during anaerobic activities are related to the effect of caffeine in some portion of the central nervous system (CNS), the propagation of neural signs between brain and neuromuscular junction, and also to the effect of caffeine on the skeletal muscle, facilitating the stimulation-contraction of the skeletal muscle. Some studies have been indicating increases of muscular strength accompanied by greater resistance to the installation of the process of muscular fatigue after caffeine ingestion. It has been suggested that it happens much more by the direct action of caffeine in CNS than by its action at peripherical level. Regarding maximum and submaximum exercises of short duration, the studies have been controversial, although most of them indicates that caffeine seems to improve the performance significantly in maximum exercises of short duration ( $<5 \mathrm{~min})$, when not preceded by sub maximum prolonged exercises. However, these results need to be confirmed, as well as the mechanisms of action of caffeine in these types of efforts.

UNITERMS: Caffeine. Ergogenic. Performance. Anaerobic exercise.

\section{AGRADECIMENTOS}

Os autores agradecem à FAPESP e à CAPES pelo apoio financeiro e bolsas outorgadas.

\section{REFERÊNCIAS BIBLIOGRÁFICAS}

ALTIMARI, L.R.; CYRINO, E.S.; ZUCAS, S.M.; BURINI, R.C. Efeitos ergogênicos da cafeína sobre o desempenho físico. Paul. J. Phys. Educ., v.14, n.2, p.141-158, 2000.

ALTIMARI, L.R.; CYRINO, E.S.; ZUCAS, S.M.; OKANO, A.H.; BURINI, R.C. Cafeína: ergogênico nutricional no esporte. Braz. J. Sci. Mov., v.9, n.3, p.5764, 2001.

ANDERSON, M.E.; BRUCE, C.R.; FRASER, S.F.; STEPTO, N.K.; KLEIN, R.; HOPKINS, W.G.; HAWLEY, J.A. Improved 2000-meter rowing performance in competitive oarswomen after caffeine ingestion. Int. J. Sport. Nutr. Exerc. Metab., v.10, n.4, p.464-475, 2000 .

ANSELME, F.; COLLOMP, K.; MERCIER, B.; AHMAIDI, S.; PREFAUT, C. Caffeine increases maximal anaerobic power and blood lactate concentration. Eur. J. Appl. Physiol., v.65, n.2, p.188191, 1992.

BARONE, J.J.; ROBERTS, H.R. Caffeine consumption. $F d$. Chem. Tox., v.34, n.1, p.119-129,1996.

BELL, D.G.; JACOBS, I.; ELLERINGTON, K. Effect of caffeine and ephedrine ingestion on anaerobic exercise performance. Med. Sci. Sports Exerc., v.33, n.11, p.1399-1403, 2001.

BOND, V.; GRESHAM, K.; MCRAE, J.; TEARNEY, R.J. Caffeine ingestion and isokinetic strength. Br. J. Sports Med., v.20, n.3, p.135-137, 1986.

BRAGA, L.C.; ALVES, M.P. A Cafeína como recurso ergogênico nos exercícios de endurance. Braz. J. Sci. Mov., v.8, n.3, p.33-37, 2000.

BRUCE, C.R.; ANDERSON, M.E.; FRASER, S.F.; STEPTO, N.K.; KLEIN, R.; HOPKINS, W.G.; HAWLEY, J.A. Enhancement of 2000-m rowing performance after caffeine ingestion. Med. Sci. Sports Exerc., v.32, n.11, p.1958-1963, 2000. 
BUCCI, L.R. Selected herbals and human exercise performance. Am. J. Clin. Nutr., v.72, n.2(suppl), p.624S-636S, 2000.

CLARKSON, P.M. Nutritional ergogenic aids: caffeine. Int. J. Sports Nutr., v.3, n.1, p.103-111, 1993.

COLLOMP, K.; CAILlAUd, C.; AUDRAM, M.; CHANAL, J.L.; PREFAUT, C. Effect of acute or chronic administration of caffeine on performance and on catecholamines during maximal cycle ergometer exercise. C. R. Seances Soc. Biol. Fil., v.184, n.1, p.87-92, 1990.

COLLOMP, K.; AHMAIDI, S.; AUDRAN, M.; CHANAL, J.L.; PREFAUT, C. Effects of caffeine ingestion on performance and anaerobic metabolism during the Wingate test. Int. J. Sports Med., v.12, n.5, p.439-443, 1991.

COLLOMP, K.; AHMAIDI, S.; CHATARD, J.C.; AUDRAN, M.; PREFAUT, C. Benefits of caffeine ingestion on sprint performance in trained and untrained swimmers. Eur. J. Appl. Physiol., v.64, n.4, p.377-380, 1992.

COYLE, E.F. Fluid and fuel intake during exercise. J. Sports Sci., v.22, n.1, p.39-55, 2004.

DAVIS, J.M.; BAILEY, S.P. Possible mechanisms of central nervous system fatigue during exercise. Med. Sci. Sports Exerc., v.29, n.1, p.45-57, 1997.

DAVIS, J.M.; ZHAO, Z.; STOCK, H.S.; MEHL, K.A.; BUGGY, J.; HAND, G.A. Central nervous system effects of caffeine and adenosine on fatigue. Am. J. Physiol. Regul. Integr. Comp. Physiol., v.284, n.2, p.399-404, 2003.

DOHERTY, M. The effects of caffeine on the maximal accumulated oxygen deficit and short-term running performance. Int. J. Sports Nutr., v.8, n.2, p.95-104, 1998.

DOHERTY, M.; SMITH, P.M.; DAVISON, R.C.; HUGHES, M.G. Caffeine is ergogenic after supplementation of oral creatine monohydrate. Med. Sci. Sports Exerc., v.34, n.11, p.1785-1792, 2002.

DUTHEL, J.M.; VALLON, J.J.; MARTIN, G.; FERRET, J.M.; MATHIEU, R.; VIDEMAN, R. Caffeine and sport: role of physical exercise. Med. Sci. Sports Exerc., v.23, n11,p.980-985, 1991.
ELLENHORN'S MEDICAL TOXICOLOGY. Diagnosis and Treatment of Human Poisoning. ${ }^{\text {th }}$ ed. Baltimore: Williams \& Wilkins,1997, p. 356.

FALK, B.; BURSTEIN, R.; ASHILENAZI, I.; SPILBERG, O.; ALTER, J.; ZYLBER-KATZ, E.; RUBINSTEIN, A.; BASHAN, N.; SHAPIRO, Y. The effect of caffeine ingestion on physical performance after prolonged exercise. Eur. J. Appl. Physiol., v.59, n.3, p.168-173, 1989.

FERDHOLM, B.B. On the mechanism of action of theophilline and caffeine. Acta Med. Scand., v.217, n1, p.149-153, 1985.

FITTS, R.H. Cellular mechanisms of fatigue muscle. Physiol. Rev., v.74, n.1, p.49-93, 1994.

GEORGE, A.J. Central nervous system stimulants. Baillieres Best. Pract. Res. Clin. Endocrinol. Metab., v.14, n.1, p.79-88, 2000.

GOABDUFF, T.; DREANO, Y.; GUILOIS, B.; MENEZ, J.F.; BERTHOU, F. Induction of liver and kidney CYP 1A1/1A2 by caffeine in rat. Biochem. Pharmacol., v.52, n.9, p.1915-1919, 1986.

GOMES, M.R.; TIRAPEGUI, J. Relação de alguns suplementos nutricionais e o desempenho físico. Arch. Latinoam. Nutr., v.50, n.4, p.317-329, 2000.

GRAHAM, T.E. Caffeine and exercise: metabolism, endurance and performance. Sports Med., v.31, n.11, p.785-807, 2001a.

GRAHAM, T.E. Caffeine, coffee and ephedrine: impact on exercise performance and metabolism. Can. J. Appl. Physiol., v.26, n.1 (Suppl), p.103S-119S, 2001b.

GRAHAM, T.E.; RUSH, J.W.; VAN SOEREN, M.H. Caffeine and exercise: metabolism and performance. Can. J. Appl. Physiol., v.19, n.2, p.111-138, 1994.

GREER, F.; MCLEAN, C.; GRAHAM, T.E. Caffeine, performance and metabolism during repeated Wingate exercise tests. J. Appl. Physiol., 85: 1502-1508, 1998.

HARLAND, B.F. Caffeine and nutrition. Nutrition, v.16, n.7-8, p.522-526, 2000. 
HESPEL, P.; OP'T EIJNDE, B.; VAN LEEMPUTTE, M. Opposite actions of caffeine and creatine on muscle relaxation time in humans. J. Appl. Physiol., v.92, n.2, p.513-518, 2002.

ISSEKUTZ, B.J.R. Effect of beta-adrenergic blockade on lactate turnover in exercising dogs. J. Appl. Physiol., v.57, n.6, p.1754-1759, 1984.

JACKMAN, M.; WENDLING, A.; FRIARS, D.; GRAHAM, T.E. Metabolic, catecholamine, and endurance responses to caffeine during intense exercise. J. Appl. Physiol., v.81,n.4, p.1658-1663, 1996.

JUHN, M.S. Ergogenic aids in aerobic activity. Curr. Sports Med. Rep., v.1, n.4, p.233-238, 2002.

JUHN, M.S. Popular sports supplements and ergogenic aids. Sports Med., v.33, n.12, p.921-939, 2003.

KALMAR, J.M.; CAFARELLI, E. Effects of caffeine on neuromuscular function. J. Appl. Physiol., v.87, n.2, p.801-808, 1999.

KALOW, W.; TANG, B. The use of caffeine for enzymatic assays: A critical appraisal. Clin. Pharmacol. Ther., v.53, n.3, p.503-514, 1993.

LOPES, J.M.; AUBIER, M.; JARDIM, J.; ARANDA, J.V.; MACKLEM, P.T. Effect of caffeine on skeletal muscle function before and after fatigue. J. Appl. Physiol., v.54, n.5, p.1303-1305, 1983.

McLEAN, C.; GRAHAM, T.E. The impact of gender and exercise on caffeine pharmacokinetics. Med. Sci. Sports Exerc., v.30, n.5 (suppl), p.S243, 1998.

MAUGHAN, R.J. Nutritional ergogenic aids and exercise performance. Nutr. Res. Rev., v.12, n.1, p.255-280, 1999.

MAUGHAN, R. The athlete's diet: nutritional goals and dietary strategies. Proc. Nutr. Soc., v.61, n.1, p.87-96, 2002.

MAUGHAN, R.J.; KING, D.S.; LEA, T. Dietary supplements. J. Sports Sci., v.22, n.1, p.95-113, 2004.

PAGALA, M.K.; TAYLOR, S.R. Imaging caffeine induced $\mathrm{Ca}^{2+}$ transients in individual fast-twitch and slow-twitch rat skeletal muscle fibers. Am. J. Physiol., v.274, n.3, p.623$632,1998$.
PALUSKA, S.A. Caffeine and exercise. Curr. Sports Med. Rep., v.2, n.4, p.213-219, 2003.

PÁSCOA, M.R.S.; ALVIM, C.R.; RODRIGUES, L.O.C. Efeitos da cafeína sobre a força muscular. Min. J. Phys. Educ., v.2, n.1 (suppl), p.S56, 1994.

PATON, C.D.; HOPKINS, W.G.; VOLLEBREGT, L. Little effect of caffeine ingestion on repeated sprints in teamsport athletes. Med. Sci. Sports Exerc., v.33, n.5, p.822$825,2001$.

PINTO, S.; TARNOPOLSKY, M. Neuromuscular effects of caffeine in males and females. Can. J. Appl. Physiol., v.22, n.1(suppl), p.S48, 1997.

PIPE, A.; AYOTTE, C. Nutritional supplements and doping. Clin. J. Sports Med., v.12, n.4, p.245-249, 2002.

PLASKETT, C.J.; CAFARELLI, E. Caffeine increases endurance and attenuates force sensation during submaximal isometric contractions. J. Appl. Physiol., v.91, n.4, p.1535-1544, 2001.

RACHIMA-MAOZ, C.; PELEG, E.; ROSENTHAL, T. The effects of caffeine on ambulatory blood pressure in hypertensive patients. Am. J. Hypertens., v.11, n.8, p.1426-1432, 1998.

ROSSI, L.; TIRAPEGUI, J. Aspectos atuais sobre exercício físico, fadiga e nutrição. Rev. Paul. Educ. Fis., v.13, n.1, p.67-82, 1999.

ROY, B.; TARNOPOLSKY, M.; MACDOUGALL, J.D.; HICKS, A. Caffeine and neuromuscular fatigue in endurance athletes. Can. J. Appl. Physiol., v.19, n.1(Suppl), p.S41, 1994.

SLAVIN, N.; JOENSEN, H.K. Caffeine and Sport Performance. Phys. Sports Med., v.13, p.191-193, 1995.

SINCLAIR, C.J.D.; GEIGER, J.D. Caffeine use in sports. A pharmacological review. J. Sports Med. Phys. Fitness., v.40, n.1, p.71-79, 2000.

SPRIET, L.S. Caffeine and performance. Int. J. Sports Nutr., v.5, n.1(suppl), p.S84-99, 1995.

SPRIET, L.L.; GIBALA, M.J. Nutritional strategies to influence adaptations to training. Sports Sci., v.22, n.1, p.127-141, 2004. 
VAN NIEUWENHOVEN, M.A.; BRUMMER, R.J.M.; BROUNS, F. Gastrointestinal function during exercise: comparison of water, sport drink, and sports drink with caffeine. J. Appl. Physiol., v.89, n.3, p.1079-1085, 2000.

VANAKOSKI, J.; KOSUNEN, V.; MERIRINNE, E.; SEPPALA, T. Creatine and caffeine in anaerobic and aerobic exercise: effects on physical performance and pharmacokinetic considerations. Int. J. Clin. Pharmacol. Ther., v.36, n.5, p.258-262, 1998.

WADA. World Anti Doping Agency. The 2004 prohibited list international standard. Disponível em: http://www.wadaama.org/en/t1 .asp. Acesso em: 11 dezembro 2004.

WALTON, C.; KALMAR, J.M.; CAFARELLI, E. Effect of caffeine on self-sustained firing in human motor units. $J$. Physiol., v.545, n.2, p.671-679, 2002.

WANG, Y.; LAU, C.E. Caffeine has similar pharmacokinetics and behavioral effects via the i.p. and p.o. routes of administration. Pharmacol. Biochem. Behav., v.60, n.1, p.271-278, 1998.
WEMPLE, R.D.; LAMB, D.R.; MCKEEVER, K.H. Caffeine vs caffeine-free sport drinks: effects on urine production at rest and during prolonged exercise. Int. $J$. Sports Med., v.18, n.1, p.40-46, 1997.

WILES, J.D.; BIRD, S.R.; HOPKINS, J.; RILEY, M. Effect of caffeinated coffee on running speed, respiratory factors, blood lactate and perceived exertion during 1500$\mathrm{m}$ treadmill running. Br. J. Sports Med., v.26, n.2, p.116120, 1992.

WILLIAMS, J.H.; SIGNORILE, J.F.; BARNES, W.S.; HENRICH, T.W. Caffeine, maximal power output and fatigue. Br. J. Sports Med., v.22, n.4, p.132-134, 1988.

WYSS, V.; GRIBANDO, C.; GANZIT, G.P.; RIENZI, A.; SPERONE, G Influenza di prodotti caffeinici sulla potenza e sulla capacità anaerobiche in soggetti giovani. Méd. Del. Sport., v.39, n.3, p.467-476, 1986.

YAMADA, Y.; NAKAZATO, Y.; OHGA, A. The mode of action of caffeine on catecholamine release from perfused adrenal glands of cat. Br. J. Pharmacol., v.98, n.2, p.351$356,1989$.

Recebido para publicação em 22 de janeiro de 2005 Aceito para publicação em 13 de abril de 2005 